\title{
A multi-criteria approach to fair and efficient bandwidth allocation
}

\author{
Włodzimierz Ogryczak ${ }^{\mathrm{a}, *}$, Adam Wierzbicki $^{\mathrm{b}}$, Marcin Milewski $^{\mathrm{a}}$ \\ ${ }^{a}$ Faculty of Electronics and IT, Warsaw University of Technology, 00-665 Warsaw, Poland \\ ${ }^{\mathrm{b}}$ Polish-Japanese Institute of Information Technology, 02-008 Warsaw, Poland
}

Received 4 February 2005; accepted 11 December 2005

Available online 13 November 2006

\begin{abstract}
In systems which serve many users there is a need to respect some fairness rules while looking for the overall efficiency. This applies among others to network design where a central issue is how to allocate bandwidth to flows efficiently and fairly. The so-called max-min fairness is widely used to meet these goals. However, allocating the bandwidth to optimize the worst performance may cause a large worsening of the overall throughput of the network. In this paper we show how the concepts of mult-criteria equitable optimization can effectively be used to generate various fair and efficient allocation schemes. We introduce a multi-criteria model equivalent to equitable optimization and we develop a corresponding reference point procedure to generate fair and efficient bandwidth allocations. Our analysis is focused on the nominal network design for elastic traffic that is currently the most significant traffic of IP networks. The procedure is tested on a sample network dimensioning problem for elastic traffic and its abilities to model various preferences are demonstrated.
\end{abstract}

(C) 2006 Elsevier Ltd. All rights reserved.

Keywords: Multicriteria; Fairness; Telecommunications; Resource allocation

\section{Introduction}

Resource allocation decisions are usually concerned with the allocation of limited resources so as to achieve the best system performance. However, in networking there is a need to respect some fairness rules while looking for the overall efficiency. A fair way of distribution of the bandwidth (or other network resources) among competing demands becomes a key issue in computer networks [1] and telecommunication network design in general $[2,3]$. This paper deals with problems of

\footnotetext{
This paper was processed by Guest Editors Margaret M. Wiecek, Matthias Ehrgott, Georges Fadel and José Rui Figueira.

* Corresponding author. Tel.: +48226607750; fax: +48228253719 .

E-mail address: ogryczak@ia.pw.edu.pl (W. Ogryczak).
}

bandwidth allocation within telecommunication networks. Therefore, we focus on the approaches that attempt to provide a fair (equal) treatment of all the activities (demands) [4,5] while allocating resources.

Expanding demand on the Internet services has led to an increased role of the traffic carried by the IP protocol in telecommunication networks. The TCP protocol is the most frequently used transport protocol in besteffort IP networks. The data traffic carried by the TCP protocol adapts its throughput to the amount of available bandwidth. Such traffic, called elastic traffic, is capable to use the entire available bandwidth, but it is also able to reduce its throughput in the presence of contending traffic. It should be noted here that elastic traffic communicated by the TCP protocol is currently the most significant portion of traffic in IP networks. Applications such as World Wide Web, e-mail, or Peer-to-Peer 
file-sharing all use the TCP protocol and therefore communicate elastic traffic, which forms the majority of the traffic volume in IP networks. Nowadays, network management often faces the problem of designing networks that carry elastic traffic. These network design problems are essentially network dimensioning problems as they can be reduced to a decision about link capacities. Flow sizes are outcomes of the design problem, since the flows adapt to given network resources on a chosen path.

Network management must stay within a budget constraint on link bandwidth to expand network capacities. An obvious goal is to achieve a high throughput of the IP network to increase the multiplexing gains (due to the use of packet switching by the IP protocol). This traffic is offered only a best-effort service, and therefore network management is not concerned with offering guaranteed levels of bandwidth to the traffic. A straightforward network dimensioning with elastic traffic could be thought of as a search for such network flows that will maximize the aggregate network throughput while staying within a budget constraint for the costs of link bandwidth. However, maximizing aggregate throughput can result in extremely unfair solutions allowing even for starvation of flows for certain services. On the other extreme, while looking at the problem from the perspective of a network user, the network flows between different nodes should be treated as fairly as possible [6]. The so-called max-min fairness (MMF) [7,8] is widely considered as such ideal fairness criteria. Indeed, the lexicographic max-min optimization used in the MMF approach generalizes equal sharing at a single link bandwidth to any network while maintaining the Pareto optimality. Certainly, allocating the bandwidth to optimize the worst performance may cause a large worsening of the overall throughput of the network. Therefore, network management must consider two conflicting goals: increasing throughput and providing fairness.

The search for compromise solutions that do not starve network flows, and give satisfying levels of throughput has led to the development of methods depending on maximization of the sum of the flows evaluated with some (concave) utility function. In particular, the so-called proportional fairness (PF) approach [9] maximizes the sum of logarithms of the flows. The approach has been further extended to a parametric class of concave utility functions [10]. However, every such approach requires to build (or to guess) a utility function prior to the analysis and later it gives only one possible compromise solution. More general parametric approach may depend on the use of the socalled ordered weighted averaging (OWA) with weights assigned to the ordered outcomes (flows) thus allowing to model various fair preferences [11].

It is very difficult to identify and formalize the preferences at the beginning of the decision process. Therefore, a decision support process is usually needed that attempts to gain additional preference information interactively, simultaneously allowing the decision-maker (DM) to learn the problem during the process with possibly evolving preferences. This can be effectively achieved with the so-called quasi-satisficing approach to multi-criteria decision problems [12]. The best formalization of the quasi-satisficing approach to multi-criteria optimization has been proposed and developed mainly by Wierzbicki [13] as the reference point method. The reference point method (RPM) is an interactive technique where the DM specifies preferences in terms of aspiration levels (reference point), i.e. by introducing desired (acceptable) levels for several criteria.

The purpose of this work is to show a multi-criteria model that allows to represent consistently the overall efficiency and fairness goals and thereby to apply the reference point methodology to the bandwidth allocation problems effectively. The paper is organized as follows. In the next section, we formalize the considered network dimensioning problem. In Section 3, basic fair solution concepts for resource allocation are related to the multi-criteria equitable optimization theory. In Section 4, the reference point methodology is applied to the multi-criteria which allows us to model various fair and efficient allocation schemes with simple control parameters. Finally, in Section 5 we present some results of our initial computational experience with this new approach.

\section{The bandwidth allocation problem}

The basic problem of network dimensioning with elastic traffic can be formulated as a linear programming (LP) based resource allocation model as follows [2]. Given a network topology $G=\langle V, E\rangle$, consider a set of pairs of nodes as set $I=\{1,2, \ldots, m\}$ of services representing the elastic flow from source $v_{i}^{s}$ to destination $v_{i}^{d}$. For each service, we have a given set $P_{i}$ of possible routing paths in the network from the source to the destination. This information can be summarized with binary coefficients $\delta_{\text {eip }}$, where $\delta_{\text {eip }}=1$, if link $e$ belongs to routing path $p \in P_{i}$ (connecting $v_{i}^{s}$ with $v_{i}^{d}$ ), and $\delta_{\text {eip }}=0$ otherwise.

For each service $i \in I$, the elastic flow from source $v_{i}^{s}$ to destination $v_{i}^{d}$ is a variable representing the model outcome and it will be denoted by $x_{i}$. This flow may be realized along various paths $p \in P_{i}$. The flow may be 
either split among several paths or a single path must be finally selected to serve the entire flow. Actually, the latter case of nonbifurcated flows is more commonly required. Both bifurcated or nonbifurcated flows may be modeled as $x_{i}=\sum_{p \in P_{i}} x_{i p}$ where $x_{i p}$ (for $p \in P_{i}$ ) are nonnegative variables representing the elastic flow from source $v_{i}^{s}$ to destination $v_{i}^{d}$ along routing path $p$. The single-path model requires additional multiple choice constraints to enforce nonbifurcated flows. This can be implemented with additional binary (flow assignment) variables $u_{i p}$ equal 1 if path $p \in P_{i}$ is assigned to serve flow $x_{i}$, and 0 otherwise. Assuming existence of some constant $M$ upper bounding the largest possible total flow $x_{i}$, the assignment variables $u_{i p}$ can easily be used to limit the number of positive flows $x_{i p}$ with the following constraints:

$$
\begin{aligned}
& 0 \leqslant x_{i p} \leqslant M u_{i p}, \quad u_{i p} \in\{0,1\} \quad \forall i \in I ; \quad p \in P_{i} \\
& \sum_{p \in P_{i}} u_{i p}=1 \quad \forall i \in I .
\end{aligned}
$$

The network dimensioning problem depends on allocating the bandwidth to several links in order to maximize flows of all the services (demands). Typically, the network is already operated and some bandwidth is already allocated (installed) while decisions are rather related to the network expansion. Therefore, we assume that each link $e \in E$ has already capacity $a_{e}$ while decision variables $\xi_{e}$ represent the bandwidth newly allocated to link $e \in E$, thus expanding the link capacity to $a_{e}+\xi_{e}$. Certainly, all the decision variables must be nonnegative: $\xi_{e} \geqslant 0$ for all $e \in E$ and there are usually some bounds (upper limits) on possible expansion of the links capacities: $\xi_{e} \leqslant \bar{a}_{e}$ for all $e \in E$. Finally, the following constraints must be fulfilled:

$$
\begin{aligned}
& \sum_{i \in I} \sum_{p \in P_{i}} \delta_{e i p} x_{i p} \leqslant a_{e}+\xi_{e} \quad \forall e \in E, \\
& 0 \leqslant \xi_{e} \leqslant \bar{a}_{e} \quad \forall e \in E \\
& \sum_{p \in P_{i}} x_{i p}=x_{i} \quad \forall i \in I,
\end{aligned}
$$

where Eqs. (5) define the total service flows, while inequalities (3) establish the relation between the service flows and the link bandwidths. The quantity $y_{e}=$ $\sum_{i \in I} \sum_{p \in P_{i}} \delta_{\text {eip }} x_{i p}$ is the load of link $e$ and it cannot exceed the available link capacity.

Further, for each link $e \in E$, the cost of allocated bandwidth is defined. In the basic model of network dimensioning it is assumed that any real amount of bandwidth may be installed and marginal costs $c_{e}$ of link bandwidth is given. Hence, the corresponding link dimensioning function expressing amount of capacity (bandwidth) necessary to meet a required link load [2] is a linear function. While allocating the bandwidth to several links in the network dimensioning process the decisions must keep the cost within available budget $B$ for all link bandwidths. Hence the following constraint must be satisfied:

$\sum_{e \in E} c_{e} \xi_{e} \leqslant B$.

The model constraints (3)-(6) together with respective nonnegativity requirements define a linear programming (LP) feasible set. It turns into mixed integer LP (MILP), however, if nonbifurcated flows are enforced with discrete constraints (1)-(2).

Link modularity (bandwidth granulation) is a common feature in communications networks [2]. Therefore, in more realistic models for each link $e \in E$ the minimum unit of bandwidth $b_{e}$ is assumed to be available for allocation (installation) and $c_{e}$ represents the corresponding unit cost. The corresponding link dimensioning function is then a stepwise function. In the case of modular links (discrete bandwidth units $b_{e}$ ), the installed capacity $\xi_{e}$ must satisfy additional equation:

$\xi_{e}=b_{e} z_{e} \quad \forall e \in E$,

where $z_{e}$ is an integer decision variable representing the number of bandwidth units installed at link $e$. The model constraints (3)-(6) extended with (7) turns then into MILP feasible set even if bifurcated flows are allowed. The network dimensioning model can be considered with various objective functions, depending on the chosen goal. One may consider two extreme approaches. The first extreme is maximization of the total throughput (the sum of flows) $\sum_{i \in I} x_{i}$. On the other extreme, the network flows between different nodes should be treated as fairly as possible which leads to maximization of the smallest flow or rather to the lexicographically expanded max-min optimization (the socalled max-min ordering) allowing also to maximize the second smallest flows provided that the smallest remain optimal, the third smallest, etc. This approach is widely recognized in networking as the so-called MMF $[7,8]$ and it is consistent with the Rawlsian theory of justice [14]. Note that for convex models there exists at least one blocked outcome which is constant on the entire set of optimal solutions to the max-min problem. Hence, the MMF solution can be found by solving a sequence of properly defined max-min problems with fixed outcomes (flows) that have been blocked by some 
critical constraints (link capacities) [15,16]. Unfortunately, in our network dimensioning model it applies only to the basic LP constraints (3)-(6). In the case of nonconvex feasible set such a blocked quantity may not exist [17], which makes the approach not applicable to the case of nonbifurcated flows enforced by discrete constraints (1)-(2).

In the simplified problem with linear link dimensioning function (no modularities) and dimensioning of a completely new network ( $a_{e}=0$ for all links), the cost of the entire path $p$ for service $i$ can be directly expressed by the formula $\kappa_{i p}=\sum_{e \in E} c_{e} \delta_{e i p}$. Hence, the cheapest path for each service can easily be identified and preselected. Constraints (6) and (3) may be then treated as equations and they allow one to eliminate variables $\xi_{e}$, thus formulating the problem as a simplified resource allocation model with only one constraint $\sum_{i=1}^{m} \kappa_{i} x_{i}=B$ and variables $x_{i}$ representing directly the decisions. In the problem under consideration the cost of available link capacity is actually nonlinear (piecewise linear) and this results in the lack of direct formula for the path cost since it depends on possible sharing with other paths of the preinstalled bandwidth (free capacity $a_{e}$ ). Such a simplification is certainly also impossible for the modular case, due to additional discrete constraints (7).

In the simplified dimensioning model (with preselected paths and continuous bandwidth) the throughput maximization approach apparently would choose one variable $x_{i}$ o which has the smallest marginal cost $\kappa_{i}^{o}=\min _{i \in I} \kappa_{i}$ and make that flow maximal within the budget limit $\left(x_{i^{o}}=B / \kappa_{i}\right.$ ) , while eliminating all other flows (lowering them to zero). On the other hand, the MMF concept applied to the simplified dimensioning model would lead us to a solution with equal values for all the flows: $x_{i}=B / \sum_{i \in I} \kappa_{i}$ for $i \in I$. Such allocating the resources to optimize the worst performance may cause a large worsening of the overall (mean) performance as the MMF throughput $\left(m B / \sum_{i \in I} \kappa_{i}\right)$ might be considerably smaller than the maximal throughput $\left(B / \min _{i \in I} \kappa_{i}\right)$. In more realistic dimensioning models assuming bandwidth modularity or other nonlinearities in link dimensioning function (like the existence of a free capacity $a_{e}$ of preinstalled bandwidth) and nonbifurcation requirements, a direct formula for the path cost is not available and the corresponding solutions are not so clear. Nevertheless, the main weaknesses of the above solutions remain valid. The throughput maximization can always result in extremely unfair solutions allowing even for starvation of certain flows while the MMF solution may cause a large worsening of the network throughput. In an example built on the backbone network of a Polish ISP, it turned out that the throughput in a perfectly fair solution could be less than $50 \%$ of the maximal throughput [11].

Network management may be interested in seeking a compromise between the two extreme approaches discussed above. One of the possible solutions depends on maximization of the sum of flows evaluated with some (concave) utility function $U(\mathbf{x})=\sum_{i \in I} u\left(x_{i}\right)$. A parametric class of utility functions [10]:

$u\left(x_{i}, \alpha\right)= \begin{cases}x_{i}^{1-\alpha} /(1-\alpha) & \text { if } \alpha \neq 1, \\ \log \left(x_{i}\right) & \text { if } \alpha=1\end{cases}$

may be used for this purpose generating various solution concepts for $\alpha \geqslant 0$. In particular, for $\alpha=0$ one gets the throughput maximization which is the only linear criterion within the entire class. For $\alpha=1$, it represents the PF [9] that maximizes the sum of logarithms of the flows while it converges to the MMF with $\alpha$ tending to the infinity. However, every such approach requires to build (or to guess) a utility function prior to the analysis and later it gives only one possible compromise solution. It is very difficult to identify and formalize the preferences at the beginning of the decision process. Moreover, apart from the trivial case of throughput maximization all the utility functions that really take into account any fairness preferences are nonlinear. Nonlinear objective functions applied to the MILP models we consider result in computationally hard optimization problems. In the following, we shall describe an approach that allows to search for such compromise solutions with multiple linear criteria rather than nonlinear objective functions.

\section{Fair allocations and equitable efficiency}

The bandwidth allocation problem we consider may be viewed as a special case of general resource allocation problem where set $I$ of $m$ services is considered and for each service $i \in I$ its measure of realization $x_{i}$ is a function $x_{i}=f_{i}(\xi)$ of allocation pattern $\xi \in A$. This function, called the individual objective function, represents the outcome (effect) of the allocation pattern for service $i$. In applications we consider $f_{i}$ expresses the service flow and a larger value of the outcome means a better effect (higher service quality or client satisfaction). This leads us to a vector maximization problem:

$\max \left\{\left(x_{1}, x_{2}, \ldots, x_{m}\right): \mathbf{x} \in Q\right\}$,

where $Q=\left\{\left(x_{1}, \ldots, x_{m}\right): x_{i}=f_{i}(\xi)\right.$ for $\left.i \in I, \xi \in A\right\}$ denotes the attainable set for outcome vectors $\mathbf{x}$. For the network dimensioning problems we consider the set $Q$ is an MILP feasible set defined by basic constraints 
(1)-(6) with additional discrete constraints (7) in the case of modular bandwidth.

Model (9) only specifies that we are interested in maximization of all outcomes $x_{i}$ for $i \in I$. In order to make it operational, one needs to assume some solution concept specifying what it means to maximize multiple outcomes. The solution concepts are defined by properties of the corresponding preference model within the outcome space. The commonly used concept of the Pareto-optimal solutions, as feasible solutions for which one cannot improve any criterion without worsening another, depends on the rational dominance $\succcurlyeq_{r}$ which may be expressed in terms of the vector inequality: $\mathbf{x}^{\prime} \succcurlyeq_{r} \mathbf{x}^{\prime \prime}$ iff $x_{i}^{\prime} \geqslant x_{i}^{\prime \prime}$ for all $i \in I$.

In order to ensure fairness, all system entities have to be equally well provided with the system's services. This leads to concepts of fairness expressed by the equitable rational preferences $[18,19]$. First of all, the fairness requires impartiality of evaluation, thus focusing on the distribution of outcome values while ignoring their ordering. That means, in the multi-criteria problem (9) we are interested in a set of outcome values without taking into account which outcome is taking a specific value. Hence, we assume that the preference model is impartial (anonymous, symmetric). In terms of the preference relation it may be written as the following axiom:

$\left(x_{\tau(1)}, x_{\tau(2)}, \ldots, x_{\tau(m)}\right) \cong\left(x_{1}, x_{2}, \ldots, x_{m}\right)$

for any permutation $\tau$ of $I$

which means that any permuted outcome vector is indifferent in terms of the preference relation. Further, fairness requires equitability of outcomes which causes that the preference model should satisfy the (Pigou-Dalton) principle of transfers. The principle of transfers states that a transfer of any small amount from an outcome to any other relatively worse-off outcome results in a more preferred outcome vector. As a property of the preference relation it represents the following axiom:

$x_{i^{\prime}}>x_{i^{\prime \prime}} \Rightarrow \mathbf{x}-\varepsilon \mathbf{e}_{i^{\prime}}+\varepsilon \mathbf{e}_{i^{\prime \prime}} \succ \mathbf{x} \quad$ for $0<\varepsilon<x_{i^{\prime}}-x_{i^{\prime \prime}}$.

The rational preference relations satisfying additionally axioms (10) and (11) are called hereafter fair (equitable) rational preference relations. We say that outcome vector $\mathbf{x}^{\prime}$ fairly dominates $\mathbf{x}^{\prime \prime}\left(\mathbf{x}^{\prime} \succ_{e} \mathbf{x}^{\prime \prime}\right)$, iff $\mathbf{x}^{\prime} \succ \mathbf{x}^{\prime \prime}$ for all fair rational preference relations $\succcurlyeq$. In other words, $\mathbf{x}^{\prime}$ fairly dominates $\mathbf{x}^{\prime \prime}$, if there exists a finite sequence of vectors $\mathbf{x}^{j}(j=1,2, \ldots, s)$ such that $\mathbf{x}^{1}=\mathbf{x}^{\prime \prime}, \mathbf{x}^{s}=\mathbf{x}^{\prime}$ and $\mathbf{x}^{j}$ is constructed from $\mathbf{x}^{j-1}$ by application of either permutation of coordinates, equitable transfer, or increase of a coordinate. Fig. 1 presents the structure

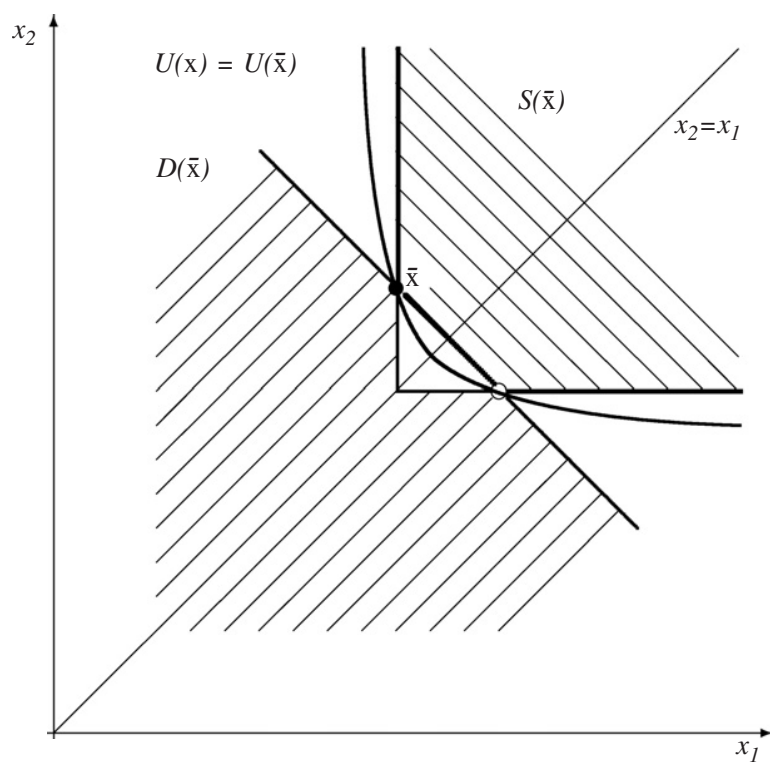

Fig. 1. Structure of the fair dominance.

of fair dominance for two-dimensional outcome vectors. For any outcome vector $\overline{\mathbf{x}}$, the fair dominance relation distinguishes set $D(\overline{\mathbf{x}})$ of dominated outcomes (obviously worse for all fair rational preferences) and set $S(\overline{\mathbf{x}})$ of dominating outcomes (obviously better for all fair rational preferences). However, some outcome vectors are left (in white areas) and they can be differently classified by various specific fair rational preferences. The MMF assigns the entire interior of the inner white triangle to the set of preferred outcomes while classifying the interior of the external open triangles as worse outcomes. Isolines of various utility functions (8) split the white areas in different ways. For instance, there is no fair dominance between vectors $(0.01,1)$ and $(0.02,0.02)$ and the MMF considers the latter as better while the proportional fairness points out the former. On theother hand, vector $(0.02,0.99)$ fairly dominates $(0.01,1)$ and all fairness models (including MMF and $\mathrm{PF})$ prefers the former.

The theory of majorization [20] includes the results which allow us to express the relation of fair (equitable) dominance as a vector inequality on the cumulative ordered outcomes [19]. This can be mathematically formalized as follows. First, introduce the ordering map $\Theta: R^{m} \rightarrow R^{m}$ such that $\Theta(\mathbf{x})=$ $\left(\theta_{1}(\mathbf{x}), \theta_{2}(\mathbf{x}), \ldots, \theta_{m}(\mathbf{x})\right)$, where $\theta_{1}(\mathbf{x}) \leqslant \theta_{2}(\mathbf{x}) \leqslant \cdots \leqslant$ $\theta_{m}(\mathbf{x})$ and there exists a permutation $\tau$ of set $I$ such that $\theta_{i}(\mathbf{x})=x_{\tau(i)}$ for $i=1, \ldots, m$. Next, apply to ordered outcomes $\Theta(\mathbf{x})$, a linear cumulative map thus resulting in the cumulative ordering map 
$\bar{\Theta}(\mathbf{x})=\left(\bar{\theta}_{1}(\mathbf{x}), \bar{\theta}_{2}(\mathbf{x}), \ldots, \bar{\theta}_{m}(\mathbf{x})\right)$ defined as

$\bar{\theta}_{i}(\mathbf{x})=\sum_{j=1}^{i} \theta_{j}(\mathbf{x}) \quad$ for $i \in I$.

The coefficients of vector $\bar{\Theta}(\mathbf{x})$ express, respectively: the smallest outcome, the total of the two smallest outcomes, the total of the three smallest outcomes, etc. The theory of majorization allow us to derive the following theorem [19].

Theorem 1. Outcome vector $\mathbf{x}^{\prime}$ fairly dominates $\mathbf{x}^{\prime \prime}$, if and only if $\bar{\theta}_{i}\left(\mathbf{x}^{\prime}\right) \geqslant \bar{\theta}_{i}\left(\mathbf{x}^{\prime \prime}\right)$ for all $i \in I$ where at least one strict inequality holds.

An allocation pattern $\xi \in A$ is called fairly (equitably) efficient or a fair solution (for short), if $\mathbf{x}=\mathbf{f}(\xi)$ is fairly nondominated. Note that each fairly efficient solution is also Pareto-optimal, but not vice verse. However, Theorem 1 permits one to express fair solutions of problem (9) as Pareto-optimal solutions to the multicriteria problem with objectives $\bar{\Theta}(\mathbf{x})$ :

$$
\max \left\{\left(\eta_{1}, \eta_{2}, \ldots, \eta_{m}\right): \eta_{k}=\bar{\theta}_{k}(\mathbf{x}) \text { for } k \in I, \mathbf{x} \in Q\right\} .
$$

For better understanding of the multi-criteria problem (13), one may consider normalized objective functions $\mu_{k}(\mathbf{x})=\bar{\theta}_{k}(\mathbf{x}) / k$ thus representing for each $k$ the mean of the $k$ smallest outcomes, called the worst conditional mean [5]. Note that the last ( $m$ th) objective in problem (13) represents the sum of outcomes thus corresponding to throughput maximization. Simple maximin optimization corresponds to maximization of the first objective in (13). The complete MMF solution concept represents the lexicographic approach to problem (13):

$\operatorname{lexmax}\left\{\left(\eta_{1}, \eta_{2}, \ldots, \eta_{m}\right): \eta_{k}=\bar{\theta}_{k}(\mathbf{x})\right.$ for $\left.k \in I, \mathbf{x} \in Q\right\}$.

While the MMF is only a specific (extreme) solution concept, the entire multi-criteria problem (13) may serve as a source of various fairly efficient allocation schemes [21]. Moreover, although the definitions of quantities $\bar{\theta}_{k}(\mathbf{x})$ are very complicated, they can be modeled with simple auxiliary constraints. Let us notice that for any given vector $\mathbf{x}$, the quantity $\bar{\theta}_{k}(\mathbf{x})$ is defined by the following LP problem:

$$
\begin{aligned}
\bar{\theta}_{k}(\mathbf{x})=\min & \sum_{i=1}^{m} x_{i} v_{i} \\
\text { s.t. } & \sum_{i=1}^{m} v_{i}=k, 0 \leqslant v_{i} \leqslant 1 \quad \text { for } i \in I .
\end{aligned}
$$

Exactly, the above problem is an LP for a given outcome vector $\mathbf{x}$ while it becomes nonlinear for a variable $\mathbf{x}$. This difficulty can be overcome by taking advantages of the LP dual to (14):

$$
\begin{aligned}
& \bar{\theta}_{k}(\mathbf{x})=\max k t-\sum_{i=1}^{m} d_{i} \\
& \text { s.t. } \quad t-x_{i} \leqslant d_{i}, d_{i} \geqslant 0 \quad \text { for } i \in I,
\end{aligned}
$$

where $t$ is an unrestricted variable while nonnegative variables $d_{i}$ represent downside deviations of outcome values $x_{i}$ from the value of $t$ [22].

Formula (15) allows us to formulate problem (13) as follows:

$$
\begin{array}{ll}
\max & \left(\eta_{1}, \eta_{2}, \ldots, \eta_{m}\right) \\
\text { s.t. } & \mathbf{x} \in Q \\
& \eta_{k}=k t_{k}-\sum_{i=1}^{m} d_{i k} \quad \text { for } k \in I, \\
& t_{k}-d_{i k} \leqslant x_{i}, d_{i k} \geqslant 0 \text { for } i, k \in I .
\end{array}
$$

Note that problem (16)-(18) adds only linear constraints to the original attainable set $Q$. Hence, for the basic network dimensioning problems with the set $Q$ defined by constraints (1)-(6), the resulting formulation (16)-(18) remains in the class of (multi-criteria) MILP. The same applies to the modular dimensioning model with additional constraints (7).

Although defined with simple linear constraints, the expanded model (16)-(18) introduces $m^{2}$ additional variables and inequalities. This may cause a serious computational burden for real-life network dimensioning problems. Note that the number of services (traffic demands) corresponds to the number of ordered pairs of network nodes which is already on the order of the square of the number of nodes $|V|$. Thus, finally the expanded multi-criteria model introduces $|V|^{4}$ variables and constraints which means polynomial but fast growth and may not be acceptable for larger networks. In order to reduce the problem size one may attempt the restrict the number of criteria in problem (13).

Let us consider a sequence of indices $K=$ $\left\{k_{1}, k_{2}, \ldots, k_{q}\right\}$, where $1=k_{1}<k_{2}<\cdots<k_{q-1}<k_{q}=$ $m$, and the corresponding restricted form of multicriteria model (13):

$$
\max \left\{\left(\eta_{k_{1}}, \eta_{k_{2}}, \ldots, \eta_{k_{q}}\right): \eta_{k}=\bar{\theta}_{k}(\mathbf{x}) \text { for } k \in K, \mathbf{x} \in Q\right\}
$$

with only $q<m$ criteria. Following Theorem 1, multicriteria model (13) allows us to generate any fairly 
efficient solution of problem (9). Reducing the number of criteria we restrict these capabilities. Nevertheless, one may still generate reasonable compromise solutions.

Theorem 2. If $\mathbf{x}^{o}$ is an efficient solution of the restricted problem (19), then it is an efficient (Paretooptimal) solution of the multi-criteria problem (9) and it can be fairly dominated only by another efficient solution $\mathbf{x}^{\prime}$ of (19) with exactly the same values of criteria: $\bar{\theta}_{k}\left(\mathbf{x}^{\prime}\right)=\bar{\theta}_{k}\left(\mathbf{x}^{o}\right)$ for all $k \in K$.

Proof. Suppose, there exists $\mathbf{x}^{\prime} \in Q$ which dominates $\mathbf{x}^{o}$. This means, $x_{i}^{\prime} \geqslant x_{i}^{o}$ for all $i \in I$ with at least one inequality strict. Hence, $\bar{\theta}_{k}\left(\mathbf{x}^{\prime}\right) \geqslant \bar{\theta}_{k}\left(\mathbf{x}^{o}\right)$ for all $k \in K$ and $\bar{\theta}_{k_{q}}\left(\mathbf{x}^{\prime}\right)>\bar{\theta}_{k_{q}}\left(\mathbf{x}^{o}\right)$ which contradicts efficiency of $\mathbf{x}^{o}$ within the restricted problem (19).

Suppose now that $\mathbf{x}^{\prime} \in Q$ fairly dominates $\mathbf{x}^{o}$. Due to Theorem 1, this means that $\bar{\theta}_{i}\left(\mathbf{x}^{\prime}\right) \geqslant \bar{\theta}_{i}\left(\mathbf{x}^{o}\right)$ for all $i \in I$ with at least one inequality strict. Hence, $\bar{\theta}_{k}\left(\mathbf{x}^{\prime}\right) \geqslant \bar{\theta}_{k}\left(\mathbf{x}^{o}\right)$ for all $k \in K$ and any strict inequality would contradict efficiency of $\mathbf{x}^{o}$ within the restricted problem (19). Thus, $\bar{\theta}_{k}\left(\mathbf{x}^{\prime}\right)=\bar{\theta}_{k}\left(\mathbf{x}^{o}\right)$ for all $k \in K$ which completes the proof.

It follows from Theorem 2 that while restricting the number of criteria in the multi-criteria model (13) we can essentially still expect reasonably fair efficient solution and only unfairness may be related to the distribution of flows within classes of skipped criteria. In other words we have guaranteed some rough fairness while it can be possibly improved by redistribution of flows within the intervals $\left(\theta_{k_{j}}(\mathbf{x}), \theta_{k_{j+1}}(\mathbf{x})\right]$ for $j=$ $1,2, \ldots, q-1$. Since the fairness preferences are usually very sensitive for the smallest flows, one may introduce a grid of criteria $1=k_{1}<k_{2}<\cdots<k_{q-1}<k_{q}=m$ which is dense for smaller indices while sparser for larger indices and expect solution offering some reasonable compromise between fairness and throughput maximization. In our computational analysis on the network with 132 elastic flows (Section 5) we have preselected 24 criteria including 12 the smallest flows. Note that any restricted model contains criteria $\bar{\theta}_{1}(\mathbf{x})=\min _{i \in I} x_{i}$ and $\bar{\theta}_{m}(\mathbf{x})=\sum_{i \in I} x_{i}$ among others. Therefore, it is more detailed than any bicriteria combination of max-min and throughput maximization.

\section{Multi-criteria analysis}

Following the equitable optimization results from the previous section, we may generate various fairly efficient bandwidth allocation patterns as efficient solutions of the multi-criteria problem:

$$
\begin{aligned}
\max & \left(\eta_{k}\right)_{k \in K} \\
\text { s.t. } & \mathbf{x} \in Q, \\
& \eta_{k}=k t_{k}-\sum_{i \in I} d_{i k} \quad \text { for } k \in K, \\
& t_{k}-d_{i k} \leqslant x_{i}, d_{i k} \geqslant 0 \quad \text { for } i \in I, \quad k \in K,
\end{aligned}
$$

where $K \subseteq I$ and attainable set $Q$ is defined by constraints (1)-(6) and possibly (7) in the case of bandwidth modularity. In the case of the complete multi-criteria model $(K=I)$, according to Theorem 1, all fairly efficient allocations can be found as efficient solutions to (20)-(22) while in the case of restricted set of criteria $K \subset I$ some minor unfairness related to the distribution of flows within classes of skipped criteria may occur (Theorem 2).

The simplest way to model a large gamut of fairly efficient allocations may depend on the use of some combinations of criteria $\left(\eta_{k}\right)_{k \in K}$. In particular, for the weighted sum with weights $w_{k}>0$

$$
\sum_{k \in K} w_{k} \eta_{k}=\sum_{k \in K} w_{k} \bar{\theta}_{k}(\mathbf{x})=\sum_{i \in I} v_{i} \theta_{i}(\mathbf{x})
$$

one apparently gets the so-called ordered weighted averaging (OWA) with weights $v_{i}=\sum_{k \in K: k \geqslant i} w_{k}(i \in I)$. If weights $v_{i}$ are strictly decreasing $\left(v_{1}>v_{2}>\cdots>v_{m}\right)$, i.e. in the case of full model $(K=I)$ and positive weights $w_{i}=v_{i-1}-v_{i}>0$, each optimal solution corresponding to the OWA maximization is a Pareto-optimal solution of (13). Hence, each optimal solution of the OWA maximization with strictly decreasing weights is a fair (fairly efficient) solution of (9). Actually, any equitable transfer (11) results in larger value of the OWA aggregation with strictly decreasing weights. On the other hand, an equitable transfer within a class of equal weights $v_{i}$ (corresponding to skipped criteria in $K \subset I$ ) does not change the value of the corresponding OWA aggregation which causes that the fairness among the OWA optimal flows within those classes may sometimes be improved. Moreover, in the case of LP models, as the simplified network dimensioning (3)-(6), every fairly efficient allocation scheme can be identified as an OWA optimal solution with appropriate strictly monotonic weights [19]. Several decreasing sequences of weights provide us with various aggregations. Indeed, our earlier experience with application of the OWA criterion to the simplified problem of network dimensioning with elastic traffic [11] showed that we were easily able to generate allocations representing the classical fairness 
models. On the other hand, in order to find a larger variety of new compromise solutions we needed to incorporate some scaling techniques originating from the reference point methodology. Better controllability and the complete parameterization of nondominated solutions even for non-convex, discrete problems can be achieved with the direct use of the reference point methodology.

The reference point method was introduced by Wierzbicki [13] and later extended leading to efficient implementations of the so-called aspiration/reservation based decision support (ARBDS) approach with many successful applications [12]. The ARBDS approach is an interactive technique allowing the DM to specify the requirements in terms of aspiration and reservation levels, i.e., by introducing acceptable and required values for several criteria. Depending on the specified aspiration and reservation levels, a special scalarizing achievement function is built which may be directly interpreted as expressing utility to be maximized. Maximization of the scalarizing achievement function generates an efficient solution to the multi-criteria problem. The solution is accepted by the DM or some modifications of the aspiration and reservation levels are introduced to continue the search for a better solution. The ARBDS approach provides a complete parameterization of the efficient set of the multi-criteria optimization problem. Hence, when applying the ARBDS methodology to the ordered cumulated criteria in (13), one may generate all (fairly) equitably efficient solutions of the original resource allocation problem (9).

While building the scalarizing achievement function the following properties of the preference model are assumed. First of all, for any individual outcome $\eta_{k}$ more is preferred to less (maximization). To meet this requirement the function must be strictly increasing with respect to each outcome. Second, a solution with all individual outcomes $\eta_{k}$ satisfying the corresponding reservation levels is preferred to any solution with at least one individual outcome worse (smaller) than its reservation level. Next, provided that all the reservation levels are satisfied, a solution with all individual outcomes $\eta_{k}$ equal to the corresponding aspiration levels is preferred to any solution with at least one individual outcome worse (smaller) than its aspiration level. That means, the scalarizing achievement function maximization must enforce reaching the reservation levels prior to further improving of criteria. In other words, the reservation levels represent some soft lower bounds on the maximized criteria. When all these lower bounds are satisfied, then the optimization process attempts to reach the aspiration levels.
The generic scalarizing achievement function takes the following form [13]:

$\sigma(\eta)=\min _{k \in K}\left\{\sigma_{k}\left(\eta_{k}\right)\right\}+\varepsilon \sum_{k \in K} \sigma_{k}\left(\eta_{k}\right)$,

where $\varepsilon$ is an arbitrary small positive number and $\sigma_{k}$, for $k \in K$, are the partial achievement functions measuring actual achievement of the individual outcome $\eta_{k}$ with respect to the corresponding aspiration and reservation levels ( $\eta_{k}^{a}$ and $\eta_{k}^{r}$, respectively). Thus, the scalarizing achievement function is, essentially, defined by the worst partial (individual) achievement but additionally regularized with the sum of all partial achievements. The regularization term is introduced only to guarantee the solution efficiency in the case when the maximization of the main term (the worst partial achievement) results in a non-unique optimal solution.

The partial achievement function $\sigma_{k}$ can be interpreted as a measure of the DM's satisfaction with the current value (outcome) of the $k$ th criterion. It is a strictly increasing function of outcome $\eta_{k}$ with value $\sigma_{k}=1$ if $\eta_{k}=\eta_{k}^{a}$, and $\sigma_{k}=0$ for $\eta_{k}=\eta_{k}^{r}$. Thus, the partial achievement functions map the outcomes values onto a normalized scale of the DM's satisfaction. Various functions can be built meeting those requirements [12]. We use the piecewise linear partial achievement function introduced in [18]. It is given by

$$
\sigma_{k}\left(\eta_{k}\right)= \begin{cases}\gamma\left(\eta_{k}-\eta_{k}^{r}\right) /\left(\eta_{k}^{a}-\eta_{k}^{r}\right) & \text { for } \eta_{k} \leqslant \eta_{k}^{r}, \\ \left(\eta_{k}-\eta_{k}^{r}\right) /\left(\eta_{k}^{a}-\eta_{k}^{r}\right) & \text { for } \eta_{k}^{r}<\eta_{k}<\eta_{k}^{a}, \\ \beta\left(\eta_{k}-\eta_{k}^{a}\right) /\left(\eta_{k}^{a}-\eta_{k}^{r}\right)+1 & \text { for } \eta_{k} \geqslant \eta_{k}^{a},\end{cases}
$$

where $\beta$ and $\gamma$ are arbitrarily defined parameters satisfying $0<\beta<1<\gamma$. This partial achievement function is strictly increasing and concave which guarantees its LP computability with respect to outcomes $\eta_{k}$.

Recall that in our model outcomes $\eta_{k}$ represent cumulative ordered flows $x_{i}$, i.e. $\eta_{k}=\sum_{i=1}^{k} \theta_{i}(\mathbf{x})$. Hence, the reference vectors (aspiration and reservation) represent, in fact, some reference distributions of outcomes (flows). Moreover, due to the cumulation of outcomes, while considering equal flows $\phi$ as a reference (aspiration or reservation) distribution, one needs to set the corresponding levels as $\eta_{k}=k \phi$. Certainly, one may specify any desired reference distribution in terms of the ordered values of the flows (quantiles in the probability language) $\phi_{1} \leqslant \phi_{2} \leqslant \cdots \leqslant \phi_{m}$ and cumulating them automatically get the reference values for outcomes $\eta_{k}$ representing the cumulated ordered flows. However, such rich modeling technique may be too complicated to control effectively the search for a compromise solution. Therefore, we rather consider to begin the search with 
a simplified approach based on the reference flow distribution given as a linear sequence $\phi_{k}=\phi_{1}(1+(k-$ 1) $r$ ) with the (relative) slope coefficient $r$ thus leading to the cumulated reference levels increasing quadratically $\bar{\theta}_{k}(\phi)=\phi_{1} k(2+(k-1) r) / 2$. Although, special meaning of the last (throughput) criterion should be rather operated independently from the others. Such an approach to control the search for a compromise fair and efficient bandwidth allocation has been confirmed by the computational experiments as described in the following section.

\section{Computational examples}

The reference distribution approach described in preceding sections have been tested on a sample network dimensioning problem with elastic traffic. Recall that in the case of elastic traffic, the network dimensioning procedure results in the capacities of links in a given network, and that the flows will adapt to the bandwidth available on links in the designed network. The input to a network dimensioning problem with elastic traffic consists of a network topology, of pairs of nodes that specify sources and destinations of flows, of sets of network paths that could be used for each flow, and of optional constraints on the capacities of links or on flow sizes. The user must also specify a budget for purchasing link capacity ( $B$ in (6)), prices of a unit of link capacity (possibly different for each link, $c_{e}$ in (6)), and may specify module sizes and prices for a link. The given network topology may contain information about preinstalled link capacities ( $a_{e}$ in (3)): the budget is then spent on additional link capacities that extend the present capacity of links.

The network topology of the presented problem (Fig. 2) is patterned after the backbone network of a Polish ISP [11]. The network consists of 12 nodes and 18 links. All links have unit costs equal to one, and the budget for link bandwidth is $B=1000$. Flows between any pair of different nodes have been considered (i.e., $144-12=132$ flows). Since all links have equal costs of one, the path cost is equal to the path length $(1,2$, 3 or 4 for the shortest paths in the example topology). For each flow, two alternative paths (the shortest and the second shortest) have been specified that could be used for transport. The entire flow had to travel along one of the paths with no splitting allowed (nonbifurcation formulation (1)-(2)). All flows are unbounded. However, it is clear that due to the budget constraint no flow can exceed $B$.

In [11] a simplified LP model has been studied without additional constraints on link capacity, with a

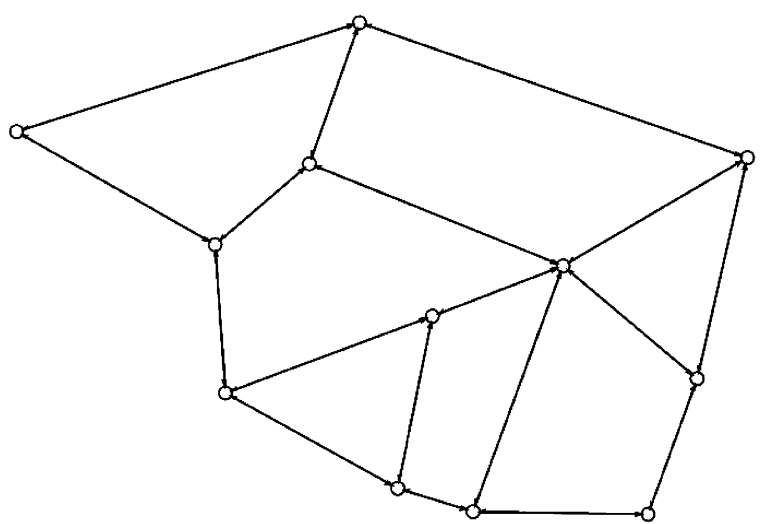

Fig. 2. Sample network topology patterned after the backbone network of Polish ISP.

limitation that flows could only use the shortest path, and with equal link costs, since in such a case it was simple to understand the best choices with respect to fairness and overall throughput. However, for such a problem it is also simple to calculate the solution obtained by the two other methods used in literature for allocation problems with fairness objectives: MMF and PF. Indeed, in [11] we have calculated these solutions and have shown that appropriate OWA aggregations allow us to obtain similar results. Additionally, using the OWA criterion, it was possible to obtain a spectrum of alternative solutions and to control the results using intuitive parameters. Here, we focus on two extensions of the problem studied in [11] that are too complex for a simple application of PF or MMF. To apply either of these methods to the discussed problem extensions, it would be necessary to solve a nonlinear optimization problem or a sequence of MILP problems with changing constraints. The proposed problem modifications also make the studied models more practical and realistic.

The first studied extension allowed flows to choose one of two paths for transport (1)-(2), added constraints that limited the capacity of certain links from above and added free link capacity for certain links (3). The intention behind the modification has been to model a situation when the network operator wishes to extend the capacity of an existing network. In this network, certain links cannot be upgraded beyond certain values due to prohibitive costs or administrative reasons (for instance, it may be cheap to use already installed fiber that has not been in use before, but it may be prohibitively expensive to install additional fiber). The existence of free link capacity and of link capacity constraints may be the reason for choosing alternative paths for certain flows. 
Additionally, a modular version of the original problem has been considered. In the second problem modification, flows were still limited to shortest paths, and no constraints on link capacities have been added. The size of a link capacity module was set to 5. For each link, integer variable $z_{e}$ has been (see (7)). Modular link capacities are frequently encountered in networks, when it is simple to upgrade a link by installing an equipment module that is capable of faster communication over the same link. Modular link capacities occur also in telecommunication networks that use traffic trunks, or portions of link capacity that are indivisible and therefore allocated in a modular way.

For all model versions, the final input to the model consisted of the reservation and aspiration levels for the sums of ordered criteria. For simplicity, all aspiration levels were set close to the optimum values of the criteria, and only reservation levels were used to control the outcome flows. One of the most significant parameters was the reservation level for the sum of all criteria (the network throughput). This value denoted by $\eta_{m}^{r}$ was selected (varying) separately from the other reservation levels. All the other reservation levels were formed following the linearly increasing sequence of the ordered values with slope (step) $r$ and where the reservation level for minimal flow was $\phi_{1}=1$. Hence, for the final criteria $\eta_{k}=\bar{\theta}_{k}(\mathbf{x})$ representing sums of the ordered outcomes in model (16)-(18), the sequence of reservation levels increased quadratically (except for the last one). Thus, the three parameters have been used to define the reference distribution but we have managed to identify various fair and efficient allocation patterns by varying only two parameters: reservation level $\eta_{m}^{r}$ for the total throughput and slope $r$ for the linearly increasing sequence.

While dealing with a simplified model in [11] we have used all criteria $\eta_{k}$ which resulted in the linear program containing a large number of constraints $\left(132^{2}\right)$. Here, we have limited the number of criteria $\eta_{k}$ to 24 , by choosing only the indices $1,2,3, \ldots, 9,10$, $11,12,18,24,30,36,48,60,72, \ldots, 120,132$ from the full set of all indices. As a result, the computation time has dropped from around one hour for each problem to the order of seconds. At the same time, the ability to control the outcomes using the reservation levels has not deteriorated; we were able to obtain similar results with the reduced set of criteria as with the full set.

In the first experiment, we have used the first model extension that introduced alternative paths for flows, free link capacity and upper limits on capacity for certain links. For certain links, free link capacity was set to values from 5 to 20, and the upper limit on the capacity of certain links was set to 20 . Due to the presence of free link capacity and upper limits on link capacity, the MILP solver found solutions where certain flows had to use alternative paths rather than shortest paths. These flows were more expensive than other flows that were allowed to use their shortest paths. Recall that we have used a single-path formulation, meaning that the entire flow had to be switched to the alternative path. Flows could not be split, which is consistent with several traffic engineering technologies used today.

In the experiment, the reservation level $\eta_{m}^{r}$ and the slope $r$ have been used to search for compromise solutions that traded off fairness against efficiency. The throughput reservation has been varied from 500 to 1100. As $\eta_{m}^{r}$ increases, the cheaper flows receive more throughput at the expense of more expensive (longer) flows. For values of $\eta_{m}^{r}$ above 1100, some flows were starved, and therefore these outcomes were not considered further.

The linear increase of the other reservation levels was varied as well. The parameter $r$ could have values of: $0.02,0.03$ and 0.04 . The results of the experiment are shown in Fig. 3 with the corresponding absolute Lorenz curves [23]. The figures present plots of cumulated ordered flows $\bar{\theta}_{k}(\mathbf{x})$ versus number $k$ (rank of a flow in ordering according to flow throughput) which means that the normalizing factor $1 / m=\frac{1}{132}$ has been ignored (for both the axes). The total network throughput is represented in the figures by the height of the right end of the curve $\left(\bar{\theta}_{132}(\mathbf{x})\right)$. A perfectly equal distribution of flows would be graphically depicted by an ascending line of constant slope. All other (unequal) distributions of flows are represented by convex lines. First of all, one may notice that all the lines intersect each other which guarantees that no solution fairly dominates any other solution (Theorem 1). This confirms that our approach enables us to generate various fair (fairly efficient) solutions. Due to the limited resources, any increase of the throughput reservation enforces increasing of the cheaper flows (implemented on shorter paths) while restricting the most expensive flows (longer paths) sharing the same link capacities. This appears with reversal order of the solution lines at their ends. Actually, it turns out that all the lines intersect each other around the same point of $k=110$. Hence, the available budget essentially limits the maximum throughput of about $80 \%$ of the smallest flows to the level of about 500 which cannot be exceeded without starvation of some flows. Nevertheless, there is still possible to increase the total throughput (of all 132 flows) while decreasing the fairness (increasing differences among flows).

Note that under moderate throughput requirements, as $r$ increases, the medium flows gain at the expense of 

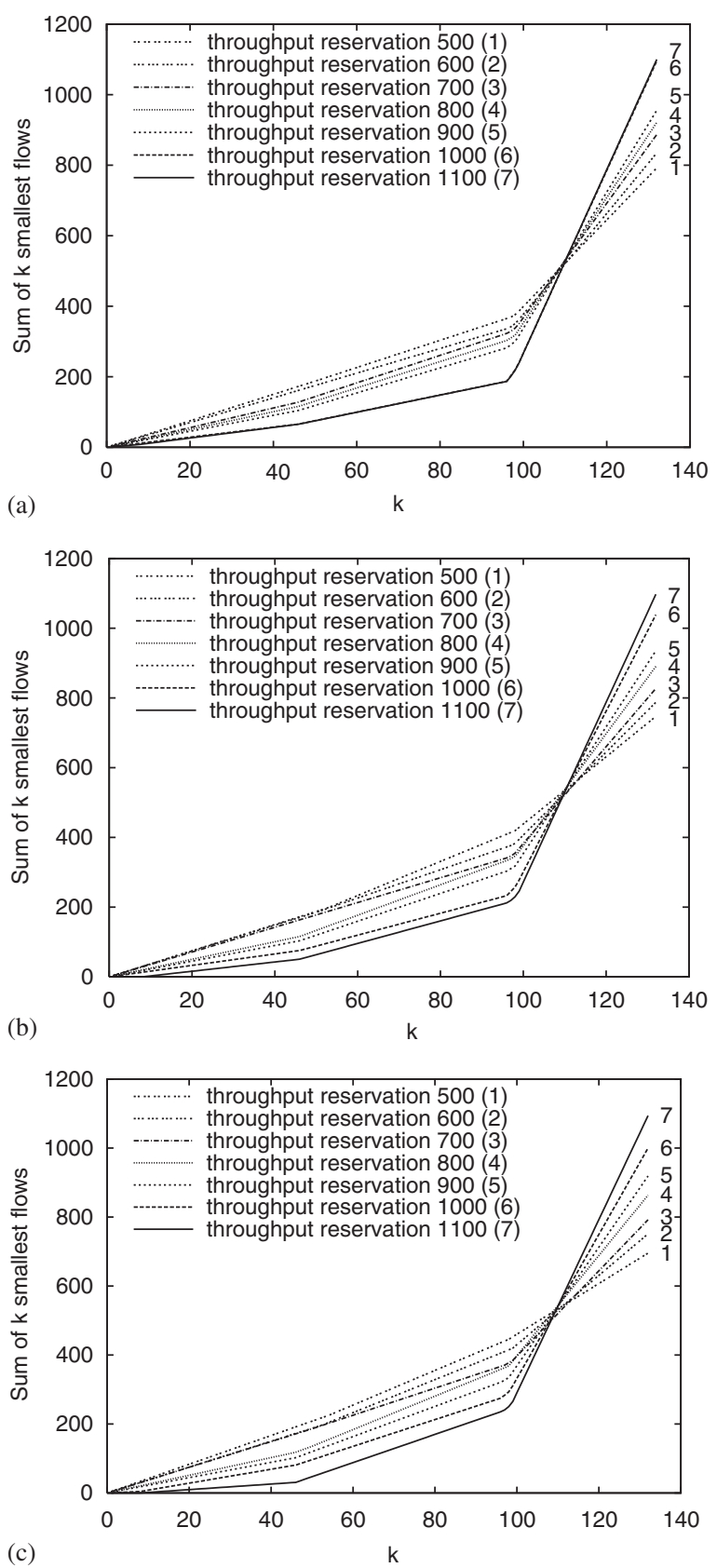

Fig. 3. Flow distribution for varying throughput reservation with $r=0.02$ (a), $r=0.03$ (b), $r=0.04$ (c).

the larger ones thus enforcing more equal distribution of flows (one may observe flattening of the curves). On the other hand, with higher throughput reservations the larger flows are protected by this requirement and increase of $r$ causes that the medium flows gain at the expense of the smallest flows (one may observe convexification of the curves). For values of $r$ higher that
0.04 , the increase of the throughput reservation resulted in flow starvation.

Observe from Fig. 3(c) that for $\eta_{m}^{r}=1100$ (and for some other values of $\eta_{m}^{r}$ ), the boundary between the largest flows (part of the Lorenz curve with the highest slope) and the second-largest flows is not sharp. The change of slope is gradual, resembling a round knee. The reason for this is the presence of three flows that should receive the same amount of throughput as the largest flows, since they are all transported on paths of the length of 1 , but cannot due to the presence of upper constraints on link capacities. These flows receive as much as they can, but some capacity is left for other flows that must travel on the same constrained link. Here the solution violates fairness in the attempt to get a higher total network throughput.

Also, note on the same figure that the boundary between the smallest flows for $\eta_{m}^{r}=500$ and for $\eta_{m}^{r}=$ 1100 is not in the same position. The reason for this is once again the upper constraint on link capacities. For $\eta_{m}^{r}=500$, there are eight flows that should be in the middle group of flows but cannot, since flows in the middle group receive so much throughput that the constraints on link capacity would be violated. Therefore, these flows are downgraded to the group of smallest flows and receive the same amount of throughput as the smallest flows-here the solution preserves fairness.

Note that the throughput reservation was effectively used to find outcomes with the desired network throughput. On the other hand, especially for large throughput reservations, the optimization procedure automatically found outcomes that divided flows into four categories according to their path costs. This shows that the presented methodology is cost-aware, and that it is possible to guarantee fairness to all flows with the same path cost (if link capacity constraints do not interfere). For the lowest throughput reservation of $\eta_{m}^{r}=500$ and $r=0.04$, the outcome was close to a perfectly fair distribution. Using the methodology described in this paper, the user can choose from a large number of different outcomes and control the trade-off between fairness and efficiency.

For the second experiment we used a slightly different dimensioning problem specification. Namely, we included the modular link capacities (7) into the model while eliminating the routing decisions by restriction of all service flows to the corresponding shortest paths. Thus, the model was still in the class of MILP but with a different discrete structure. We have repeated a search for compromise solutions using similar preference parameter configurations as in the first experiment, although taking into account the constraints on modular 


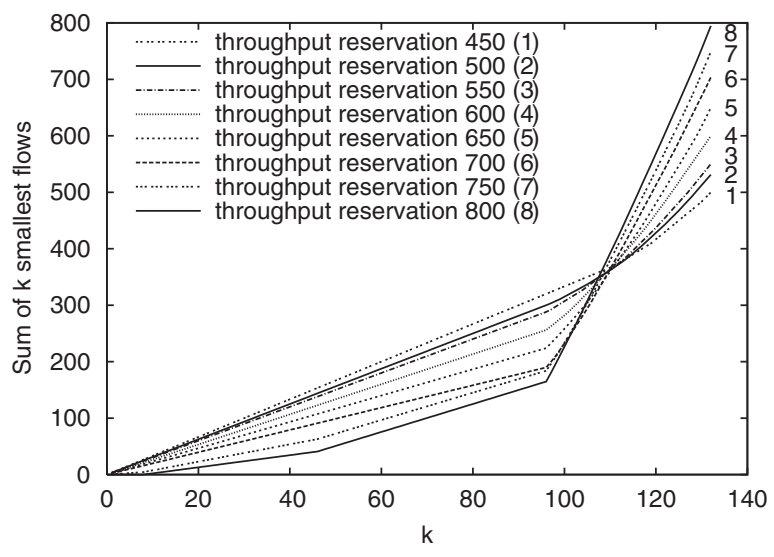

Fig. 4. Varying throughput reservation with $r=0.02$ for the model with bandwidth modularity.

bandwidth, the throughput reservation has been varied from 450 to 800 . The resulting flow distributions for the reservation slope parameter $r=0.02$ are presented in Fig. 4. Predictably, the introduction of modular link capacities makes it more difficult to find fair solutions. The outcome for $\eta_{m}^{r}=450$ is close to a perfectly even (fair) distribution, although the right end of the curve turns slightly upward. This indicates that the excess capacities of modules were used by the cheapest flows, leading to a higher network throughput than in the case of a problem without modular link capacities. On the other hand, the flows with the cheapest paths were not equal for some outcomes.

Overall, the experiments on the sample network topology demonstrated the versatility of the described methodology. The use of reservation levels, controlled by a small number of simple parameters, allowed us to search for solutions best fitted to various possible preferences of a network designer. Using an appropriate reference point procedure, one should be able to easily find a satisfactory fair and efficient allocation pattern in a few interactive steps.

\section{Concluding remarks}

A central issue in networking is how to allocate bandwidth to flows efficiently and fairly. The so-called MMF is widely used to meet these goals. Allocating the resources to optimize the worst performance may cause a large worsening of the overall (mean) performance. Therefore, several other fair allocation schemes have been sought and analyzed. Our earlier computational experiments with application of the OWA criterion to the (simplified) LP problem of network dimensioning with elastic traffic [11] showed that we were easily able to generate allocations representing the classical fairness models. On the other hand, in order to find a larger variety of new compromise solutions we needed to incorporate some scaling techniques originating from the reference point methodology. Actually it is a common flaw of the weighting approaches that they provide poor controllability of the preference modeling process and in the case of multi-criteria problems with discrete (or more general nonconvex) feasible sets, they may fail to identify several compromise efficient solutions.

In standard multi-criteria optimization, good controllability and the complete parameterization of nondominated solutions can be achieved with the direct use of the reference point methodology. While looking for fairly efficient bandwidth allocation the reference point methodology can be applied to the cumulated ordered outcomes. Our initial experiments with such an approach to the problem of network dimensioning with elastic traffic have confirmed the theoretical advantages of the method. We were able to easily generate various (compromise) fair solutions for both continuous and modular problems while controlling the search for fairly efficient compromise solutions by only two parameters. One of these parameters was a reservation level for the network throughput. The second parameter allowed the network designer to control the difference in throughputs of cheaper and more expensive flows. Still, flows with the same cost were always treated fairly. Moreover, the obtained solutions divided flows into categories determined by flow cost. For modular solutions, the cheapest flows consumed the excess link capacity. These characteristics demonstrate that the model is costaware and fulfills the axioms of equitable optimization.

\section{Acknowledgments}

The research was supported by the Ministry of Science and Information Society Technologies under Grant 3T11C 00527 (Włodzimierz Ogryczak and Adam Wierzbicki) and under Grant 3T11D 00127 (Marcin Milewski).

The authors are indebted to two anonymous referees for their helpful comments.

\section{References}

[1] Denda R, Banchs A, Effelsberg W. The fairness challenge in computer networks. Lecture notes in computer science, vol. 1922, 2000. p. 208-20.

[2] Pióro M, Medhi D. Routing, flow and capacity design in communication and computer networks. San Francisco: Morgan Kaufmann; 2004. 
[3] Tang A, Wang J, Low SH. Is fair allocation always inefficient. IEEE INFOCOM 2004.

[4] Luss H. On equitable resource allocation problems: a lexicographic minimax approach. Oper Res 1999;47:361-78.

[5] Ogryczak W, Śliwiński T. On equitable approaches to resource allocation problems: the conditional minimax solution. J Telecomm Info Tech 2002;3:40-8.

[6] Bonald T, Massoulie L. Impact of fairness on Internet performance, Proceedings of ACM Sigmetrics, June 2001. p. 82-91.

[7] Bertsekas D, Gallager R. Data networks. Englewood Cliffs: Prentice-Hall; 1987.

[8] Jaffe J. Bottleneck flow control. IEEE Trans Commun 1980;7:207-37.

[9] Kelly F, Mauloo A, Tan D. Rate control for communication networks: shadow prices, proportional fairness and stability. J Oper Res Soc 1997;49:206-17.

[10] Mo J, Walrand J. Fair end-to-end window-based congestion control. IEEE/ACM Trans Network 2000;8:556-67.

[11] Ogryczak W, Śliwiński T, Wierzbicki A. Fair resource allocation schemes and network dimensioning problems. J Telecomm Info Tech 2003;3:34-42.

[12] Wierzbicki AP, Makowski M, Wessels J. Model based decision support methodology with environmental applications. Dordrecht: Kluwer; 2000.

[13] Wierzbicki AP. A mathematical basis for satisficing decision making. Math Modelling 1982;3:391-405.
[14] Rawls J. The theory of justice. Cambridge: Harvard University Press; 1971.

[15] Klein RS, Luss H, Rothblum UG. Minimax resource allocation problems with resource-substitutions represented by graphs. Oper Res 1993;41:959-71.

[16] Marchi E, Oviedo JA. Lexicographic optimality in the multiple objective linear programming: the nucleolar solution. European J Oper Res 1992;57:355-9.

[17] Ogryczak W, Pióro M, Tomaszewski A. Telecommunication network design and max-min optimization problem. J Telecomm Info Tech 2005;3:43-56.

[18] Ogryczak W. Linear and discrete optimization with multiple criteria: preference models and applications to decision support (in Polish). Warsaw: Warsaw University Press; 1997.

[19] Kostreva MM, Ogryczak W. Linear optimization with multiple equitable criteria. RAIRO Oper Res 1999;33:275-97.

[20] Marshall AW, Olkin I. Inequalities: theory of majorization and its applications. New York: Academic Press; 1979.

[21] Ogryczak W, Wierzbicki A. On multi-criteria approaches to bandwidth allocation. Control Cybern 2004;33:427-48.

[22] Ogryczak W, Tamir A. Minimizing the sum of the $k$ largest functions in linear time. Inform Process Lett 2003;85:117-22.

[23] Kostreva MM, Ogryczak W, Wierzbicki A. Equitable aggregations and multiple criteria analysis. European J Oper Res 2004;158:362-7. 